\title{
Surface thiolation of silicon for antifouling application
}

\author{
Xiaoning Zhang ${ }^{1 *}$, Pei Gao ${ }^{2}$, Valerie Hollimon ${ }^{3}$, DaShan Brodus ${ }^{3}$, Arion Johnson ${ }^{3}$ and Hongmei Hu ${ }^{4}$
}

\begin{abstract}
Thiol groups grafted silicon surface was prepared as previously described. $1 \mathrm{H}, 1 \mathrm{H}, 2 \mathrm{H}, 2 \mathrm{H}$-perfluorodecanethiol (PFDT) molecules were then immobilized on such a surface through disulfide bonds formation. To investigate the contribution of PFDT coating to antifouling, the adhesion behaviors of Botryococcus braunii (B. braunii) and Escherichia coli (E. coli) were studied through biofouling assays in the laboratory. The representative microscope images suggest reduced B. braunii and E. coli accumulation densities on PFDT integrated silicon substrate. However, the antifouling performance of PFDT integrated silicon substrate decreased over time. By incubating the aged substrate in $10 \mathrm{mM}$ TCEP. HCl solution for $1 \mathrm{~h}$, the fouled PFDT coating could be removed as the disulfide bonds were cleaved, resulting in reduced absorption of algal cells and exposure of non-fouled silicon substrate surface. Our results indicate that the thiol-terminated substrate can be potentially useful for restoring the fouled surface, as well as maximizing the effective usage of the substrate.
\end{abstract}

Keywords: Silicon substrate, Surface thiolation, PFDT, Antifouling

\section{Introduction}

Biofouling is a complex process that involves living organisms and cells probing and attaching to surfaces. Biofouling is a big challenge for the biomedical industry because biofilms form easily on surfaces such as door handles, surgical equipment, and many other medical devices and could increase the spread of disease in humans. Data have shown that an estimated 1.7 million infections are caused from healthcare-associated infections annually [1]. In addition, because the growth of marine organisms on ship hulls can cause a drag force, biofouling can also result in decreased fuel efficiency and increased fuel consumption [2-6].

One strategy to reduce biofouling adsorption is to passivate the substrate through the coupling of antifouling molecules such as poly(ethylene glycol) [7] or poly(ethylene glycol) dimethacrylate [8]. Various works in surface modification for antifouling purposes have been summarized and reported $[9,10]$. An important

\footnotetext{
*Correspondence: XZhang@swu.edu.cn

${ }^{1}$ College of Biotechnology, Southwest University, Chongqing 400715, China

Full list of author information is available at the end of the article
}

challenge in the field of antifouling is that an antifouling coating does not last forever; it becomes less effective as it ages. It also has been brought to our attention that once the deposition of foulants has taken place, the surface modification no longer effectively prevents fouling, which is understandable considering that the effect of solute/coating interaction is severely reduced once a layer of deposited foulants is formed [10]. Therefore, once the fouling layer is formed, the old antifouling coating needs to be removed, and a new antifouling coating needs to be applied. One way of removing antifouling coating is by scraping, a time-consuming process that might damage the surface. We must therefore explore a method by which to remove the fouled coating easily.

Silicon materials are integral parts of our daily lives and have widespread applications in healthcare and manufacturing due to silicon's unique material properties, including high flexibility, chemical and thermal stability, and ease of fabrication [9]. In addition, silicon materials are mechanically and chemically resilient-able to resist wear in aqueous and organic environments-and display good electrical properties. Therefore, in this study, silicon substrate was selected as a model. Previously, we had developed a technique that allowed us to coat thiol-terminated 
silicon substrate with PFDT molecules through disulfide bonds. Here, the antifouling property of the PFDTcoated silicon substrate was tested by aging the substrate in Escherichia coli (E. coli) and Botryococcus braunii (B. braunii) cultures respectively. A large amount of B. braunii colonies were found anchored on the substrate in a 30-day immersion test. However, by applying a reducing agent, the disulfide bonds could be cleaved and the fouled coating could be removed, therefore exposing a nonfouled silicon substrate.

\section{Experimental}

\section{Chemicals and materials}

\section{Chemicals and materials for thiolation}

Anhydrous $N, N$-dimethylformamide (DMF; $\geq 99.8 \%$ ) was purchased from Fisher Scientific (United States). Anhydrous benzene ( $\geq 98 \%$ ), anhydrous alcohol ( $\leq 0.005 \%$ water), sodium hydrosulfide hydrate (pure), $1 H, 1 H, 2 H, 2 H$-perfluorodecanethiol (PFDT; 97\%), tetrabutylammonium iodide (98\%), benzoyl peroxide ( $\geq 98 \%$ ), phosphorus chloride ( $\geq 98 \%$ ), sulfuric acid (95$98 \%$ ), hydrogen peroxide (30\%, mass fraction), tris(2carboxyethyl) phosphine hydrochloride (TCEP. $\mathrm{HCl}$; $\geq 98 \%$ ), and ultraflat silicon (111) wafers (N-type) were purchased from Sigma-Aldrich (United States).

\section{Chemicals and materials for cultivation of $B$. braunii \\ and $E$. coli}

Calcium chloride dihydrate ( $\geq 99.0 \%$ ), magnesium sulfate heptahydrate $(\geq 98 \%$ ), potassium phosphate dibasic ( $\geq 98 \%$ ), potassium phosphate monobasic ( $\geq 99.0 \%$ ), and Luria-Bertani (LB) broth were purchased from Sigma-Aldrich (United States). Sodium nitrate ( $\geq 99.0 \%$ ) and sodium chloride $(\geq 99.0 \%)$ were purchased from Fisher Scientific (United States). The system used for cultivating B. braunii is equipped with a Tetra Whisper aquarium air pump (United States) to introduce air bubbles.

\section{Synthesis of thiolated silicon substrate}

The thiol-terminated silicon substrate was prepared as previously described [11]. Briefly, silicon wafers were cut into $1 \mathrm{~cm} \times 1 \mathrm{~cm}$ pieces. The wafers were cleaned with Piranha solution and were hydrogenated in $\mathrm{NH}_{4} \mathrm{~F} / \mathrm{HF}(\mathrm{aq})$ solution at room temperature. The substrates were then chlorinated in a saturated solution of $\mathrm{PCl}_{5}$ with benzyl peroxide in anhydrous benzene. Following the surface chlorination, the chlorinated substrates were placed in a $\mathrm{NaSH}$ DMF solution for surface thiolation.

\section{Preparation of PFDT modified silicon substrate}

To prepare the PFDT modified silicon surface, the thiolterminated substrate was submerged in $100 \mathrm{mmol} \mathrm{L}^{-1}$
PFDT anhydrous ethanol solution for $2 \mathrm{~h}$ immediately after the silicon surface thiolation process. Through the formation of the disulfide bonds, PFDT molecules are able to covalently bind onto the thiol-terminated silicon substrate as shown in Fig. 1. The prepared samples were then rinsed thoroughly with anhydrous ethanol, followed by sonicating in anhydrous ethanol for $5 \mathrm{~min}$. This process was repeated for several times to remove physically adsorbed PFDT molecules from sample surface. The samples were then dried with a stream of nitrogen. The attachment of PFDT molecules onto thiol-terminated silicon substrate was approved by X-ray photoelectron spectroscopy (XPS) in which a strong $\mathrm{F} 1 s$ peak was observed (Additional file 1: Figure S1).

\section{Surface characterization}

The surface chemical composition of the modified silicon substrates were characterized by a Kratos Axis Ultra DLD XPS under an ultrahigh vacuum system at a base pressure of $1.33 \times 10^{-7} \mathrm{~Pa}$ and equipped with a monochromatic $\mathrm{Al} \mathrm{K}$ alpha source. Survey spectra were obtained at a $1 \mathrm{eV}$ resolution, and high-resolution spectra were obtained at a $0.1 \mathrm{eV}$ resolution. All spectral analysis was performed with the Kratos analytical software package (Vision 2.2.10 Rev 4).

Contact angle measurements (static angles) were conducted at $25{ }^{\circ} \mathrm{C}$ with $18 \mathrm{M} \Omega \mathrm{cm}$ deionized (DI) water using a homemade experimental setup [12]. The droplet size used in contact angle measurements was $8 \mu \mathrm{L}$ so that there was no influence of gravity on contact angle measurement [13]. Water contact angles were measured from four different positions on the surface. DI water was obtained from a Millipore Direct-Q 3 water purification system.

Light micrographs of B. braunii and methylene bluestained $E$. coli were acquired by using a Nikon Eclipse $80 \mathrm{i}$ microscope (E. coli cannot be seen without staining through a microscope).

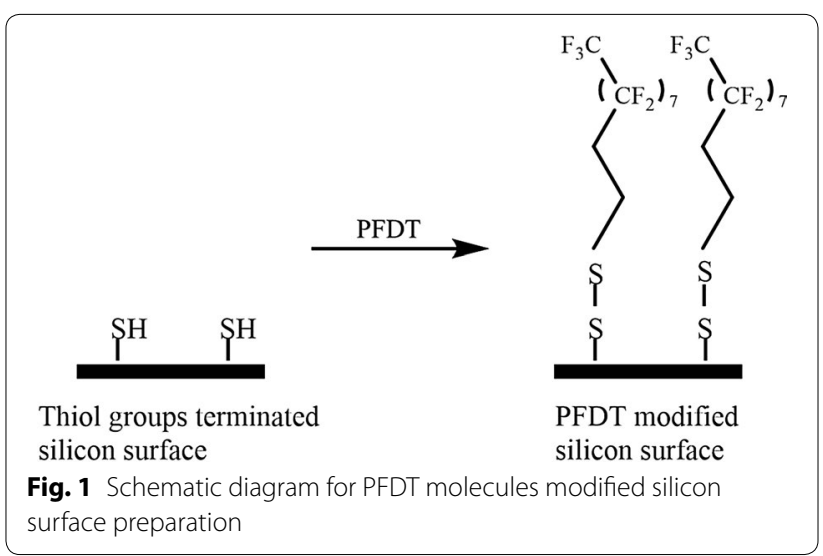




\section{Cultivation of algae}

Botryococcus braunii was obtained from the Culture Collection of Algae at the University of Texas at Austin. B. braunii was selected as a model algae cell for this study because $B$. braunii is a green microalgae widely found in temperate or tropical lakes and estuaries. The algae cells were cultivated for a period of 14 days, which is when the $B$. braunii population reached a relatively stable phase in Bristol medium (2.94 $\mathrm{mM} \mathrm{NaNO}_{3}, 0.17 \mathrm{mM}$ $\mathrm{CaCl}_{2} \cdot 2 \mathrm{H}_{2} \mathrm{O}, 0.3 \mathrm{mM} \mathrm{MgSO} \cdot \cdot 7 \mathrm{H}_{2} \mathrm{O}, 0.43 \mathrm{mM} \mathrm{K}_{2} \mathrm{HPO}_{4}$, $1.29 \mathrm{mM} \mathrm{KH}_{2} \mathrm{PO}_{4}$, and $0.43 \mathrm{mM} \mathrm{NaCl}$ ) before the antifouling assay. Cultures were grown at $19 \pm 2{ }^{\circ} \mathrm{C}$ with a 16 light/8 dark photoperiod. Lighting was supplied by a combination of warm and cold fluorescent tubes giving a luminance range of between 2200 and 2800 Lux. Continuous airflow was bubbled through the culture with a speed of $0.1 \mathrm{vvm}$, and pure carbon dioxide was supplemented to supply the carbon source every $48 \mathrm{~h}$. The in vivo absorption of the culture medium containing algal cells in each flask was monitored each day via UV-Vis spectrophotometer 2450 (Shimadzu) at $660 \mathrm{~nm}$ (Additional file 1: Figure S2).

\section{Cultivation of E. coli}

The best characterized E. coli strain, K-12 (American Type Culture Collection ATCC 25404, wild type), was provided by Dr. Yinan Wei of the University of Kentucky, Department of Chemistry, as a gift for this research. $E$. coli was maintained at $250 \mathrm{~mL}$ of Luria-Bertani (LB) broth at $37^{\circ} \mathrm{C}$, with shaking at $200 \mathrm{rpm}$ for $12 \mathrm{~h}$ until the OD600 value was approximately 0.6 . E. coli was used as a model organism because $E$. coli is a bacterium commonly found in the environment and is most widely studied.

\section{Results and discussion}

To evaluate the antifouling performance of PFDT modified silicon substrate, we immersed the PFDT-coated silicon wafer and Piranha solution (one part $98 \% \mathrm{H}_{2} \mathrm{SO}_{4}$ and two parts $30 \%$ hydrogen peroxide) cleaned silicon wafer in a B. braunii culture. As shown in Fig. 2, after culturing for 1 week, there were large amount of algal cells adhered on the Piranha solution cleaned silicon wafer (Fig. 2a), whereas fewer algal cells adhered on the PFDT modified silicon wafer (Fig. 2b). B. braunii cells on Piranha solution cleaned silicon substrate grew in small groups, and some of them formed clusters (Fig. 2a). Our observations are in agreement with the previous studies regarding the typical stages of bio-fouling development that bioorganisms can multiply locally and then assemble to form microcolonies $[14,15]$. The obvious reduction in the number of algal cells adhered to PFDT modified silicon substrate (Fig. 2b) indicates such a surface has resistance to the adhesion of algal cells, and it is not "algal friendly". This result is consistent with other published studies involving the fluorination of substrates being applied to minimize microbial adhesion [16-18].

Similar to the results obtained with B. braunii, E. coli cells readily adhere on Piranha solution cleaned silicon substrate as well (Fig. 3a). After PFDT modification, a significant reduction in the number of adherent $E$. coli cells was observed (Fig. 3b), confirming the antibacterial efficiency of the PFDT modified silicon substrate. Attached bacterial densities were calculated (Fig. 4). Five fields of view $\left(0.25 \mathrm{~mm}^{2}\right)$ on five replicate substrates were analyzed for each surface condition.

Figure 5a shows the micrograph of the PFDT modified silicon surface after 1 month of incubation in a B. braunii culture. As can be seen in the micrograph, the cell
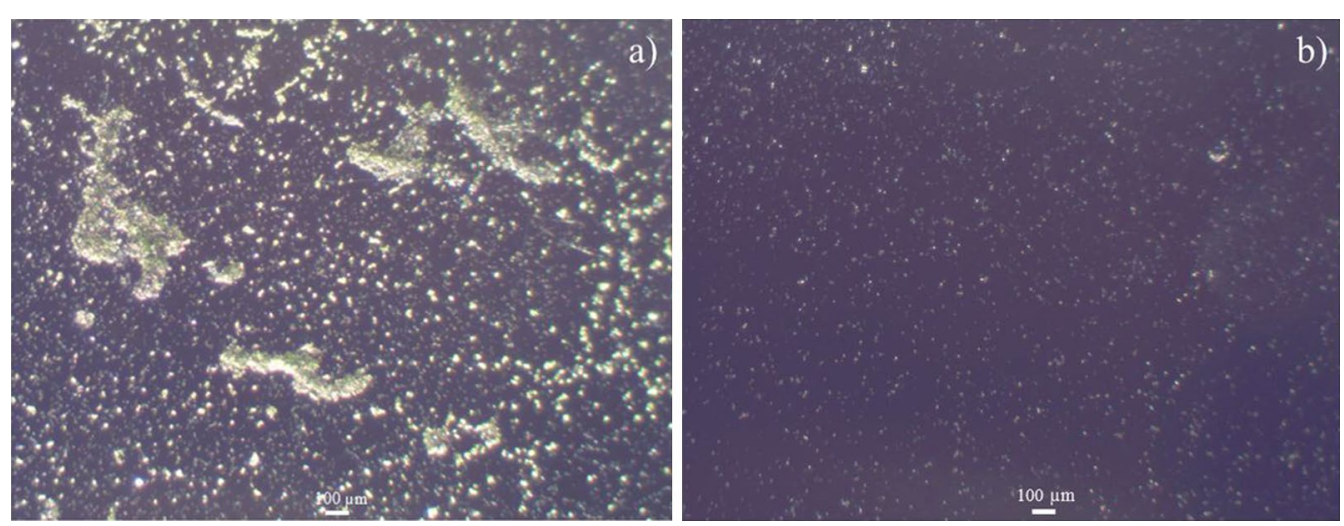

Fig. 2 Representative microscope images of Piranha solution cleaned (a) and PFDT molecules modified silicon surface (b) after immersion test in $B$. braunii culture for 1 week. The population of $B$. braunii cells attached on the Piranha solution cleaned silicon surface is much higher than that on the PFDT molecules modified silicon surface. We can therefore conclude that PFDT coated silicon surface possesses fouling resistant properties regarding B. braunii 


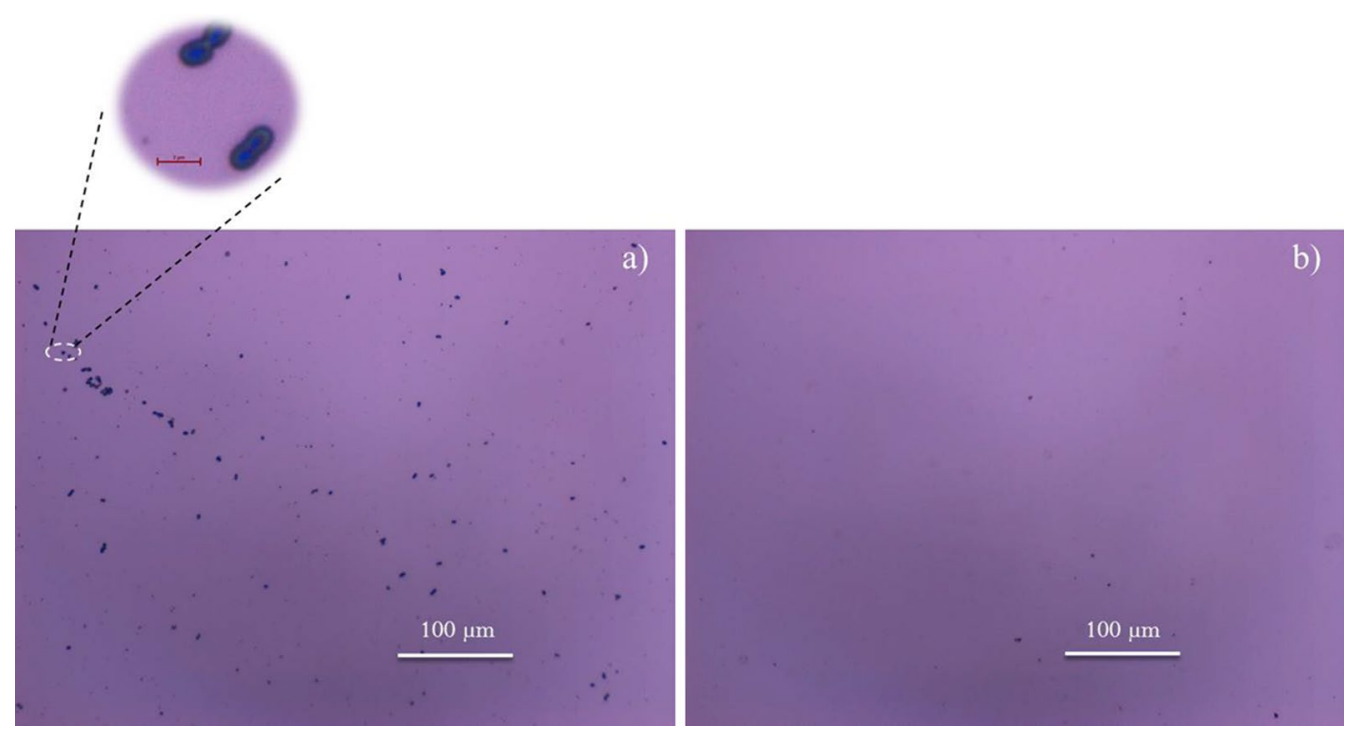

Fig. 3 Representative microscope images of Piranha solution cleaned (a) and PFDT molecules modified silicon surface (b) after immersion test in E. coli culture for $24 \mathrm{~h}$. The population of E. coli cells attached on the Piranha solution cleaned silicon surface is much higher than that on the PFDT molecules modified silicon surface. We can therefore conclude that the PFDT coated silicon surface possesses fouling resistant properties regarding E. coli (E. coli cells were stained by methylene blue)

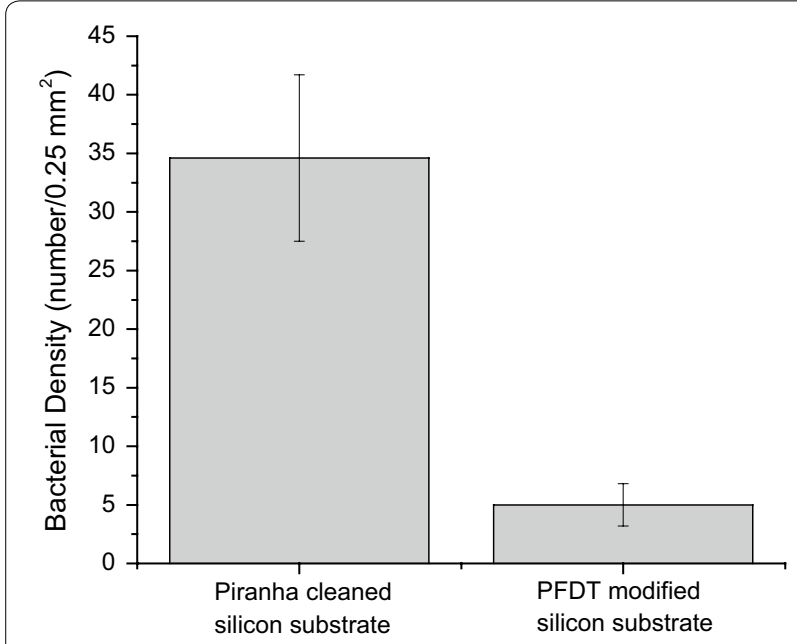

Fig. 4 Attachment of E. coli cells on Piranha solution cleaned and PFDT coated silicon surface

density, the percentage, and the average area of spread of $B$. braunii cells increased significantly throughout the test, indicating the reduced antifouling performance of PFDT modified silicon substrate. Our experimental results are in accordance with previous reports that premicrobial attachment can provide specific binding sites for further attachment of microbes and growth. The $B$. braunii microcolonies formed on the PFDT modified silicon substrate during a 1-week immersion test in order to undergo further adaption and development into B. braunii macrocolonies. However, when the sample was incubated in Bristol medium containing $10 \mathrm{mM}$ TCEP. $\mathrm{HCl}$ for $1 \mathrm{~h}$ at room temperature followed by rinsing with Bristol medium, the cell density decreased noticeably. It is because the reducing agent, TCEP. $\mathrm{HCl}$, released the PFDT layer through breaking the disulfide bond, and therefore detached the algal cells from the surface. Although several B. braunii microcolonies remained on the surface after TCEP. $\mathrm{HCl}$ treatment, it might have been due to the uneven coverage of the PFDT coating. The oxidation reduced the amount of thiol groups on the surface that could graft PFDT molecules via disulfide bonds. The attachment of B. braunii cells to the parts where no PFDT molecules were grafted could not be interrupted by TCEP. $\mathrm{HCl}$.

As a control experiment, a PFDT modified silicon substrate without $B$. braunii immersion test was submerged in $10 \mathrm{mM}$ TCEP. $\mathrm{HCl}$ solution for $1 \mathrm{~h}$ followed by rinsing with DI water, and then dried under a stream of $\mathrm{N}_{2}(\mathrm{~g})$ at room temperature. The lack of F peak in XPS survey spectra (Fig. 6) confirms that PFDT was detached from the surface after TCEP.HCl solution treatment. Peak-fitting of the $\mathrm{S} 2 s$ envelope was utilized to analyze a change in the chemical state of terminal sulfur on the silicon surface. The $\mathrm{S} 2 s$ peak instead of the $\mathrm{S} 2 p$ peak was used in this analysis because the $\mathrm{S} 2 p$ peak could avoid any possible overlap of the $\mathrm{S} 2 p(160-169 \mathrm{eV})$ region with the Si $2 s(155-165 \mathrm{eV})$ signal from the substrate [19]. It also 

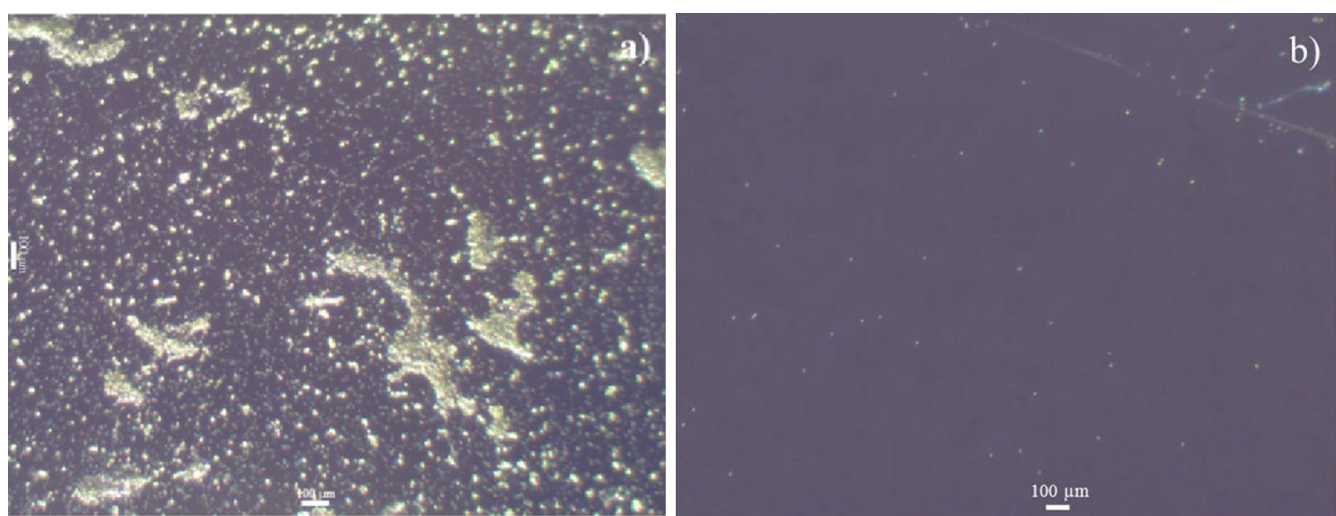

Fig. 5 Representative microscope images of PFDT molecules modified silicon surface after immersion test in B. braunii culture for 1 month at room temperature (a) and such surface after immersing in Bristol medium containing $10 \mathrm{mM} \mathrm{TCEP.HCl} \mathrm{for} 1 \mathrm{~h}$ at room temperature (b)

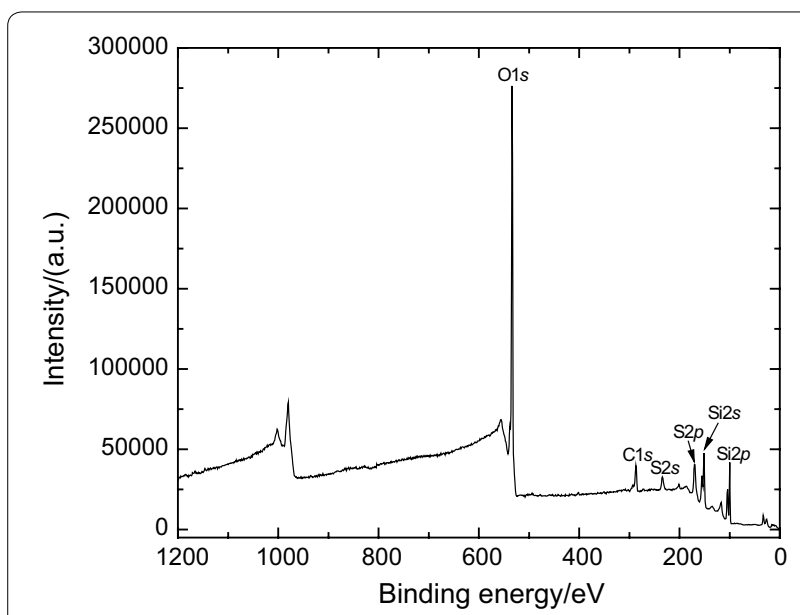

Fig. 6 XPS survey spectrum of PFDT molecules modified silicon surface after incubating in $10 \mathrm{mMTCEP} \cdot \mathrm{HCl}$ solution for $1 \mathrm{~h}$ because S $2 s$ appears as a simpler, single peak, and not a spin-orbit doublet as does S $2 p$ [20]. It was reported that the peak at nearly $(227.6 \pm 0.1) \mathrm{eV}$ was assigned to the thiol group [20]. Based on the peak areas from the deconvolution exercise (Fig. 7), thiol groups decreased from $35.3 \%$ of the surface-bound sulfur (Fig. 7a) to $1.6 \%$ (Fig. 7b). This result indicates that thiol groups released from disulfide bonds $(-\mathrm{S}-\mathrm{S}-)$ through $\mathrm{TCEP} \cdot \mathrm{HCl}$ treatment were oxidized to a significant extent.

Besides XPS, the difference in the chemical compositions was also reflected in the surface wettability of a given sample. The representative water contact angle images of chemically-modified silicon substrates in different stages are shown in Fig. 8. For the freshly prepared hydrogen-terminated silicon substrate (Fig. 8a), the water contact angle was about $78.4 \pm 1.1^{\circ}$. After thiolation, the water contact angle was about $23.6 \pm 1.2^{\circ}$ (Fig. 8b).
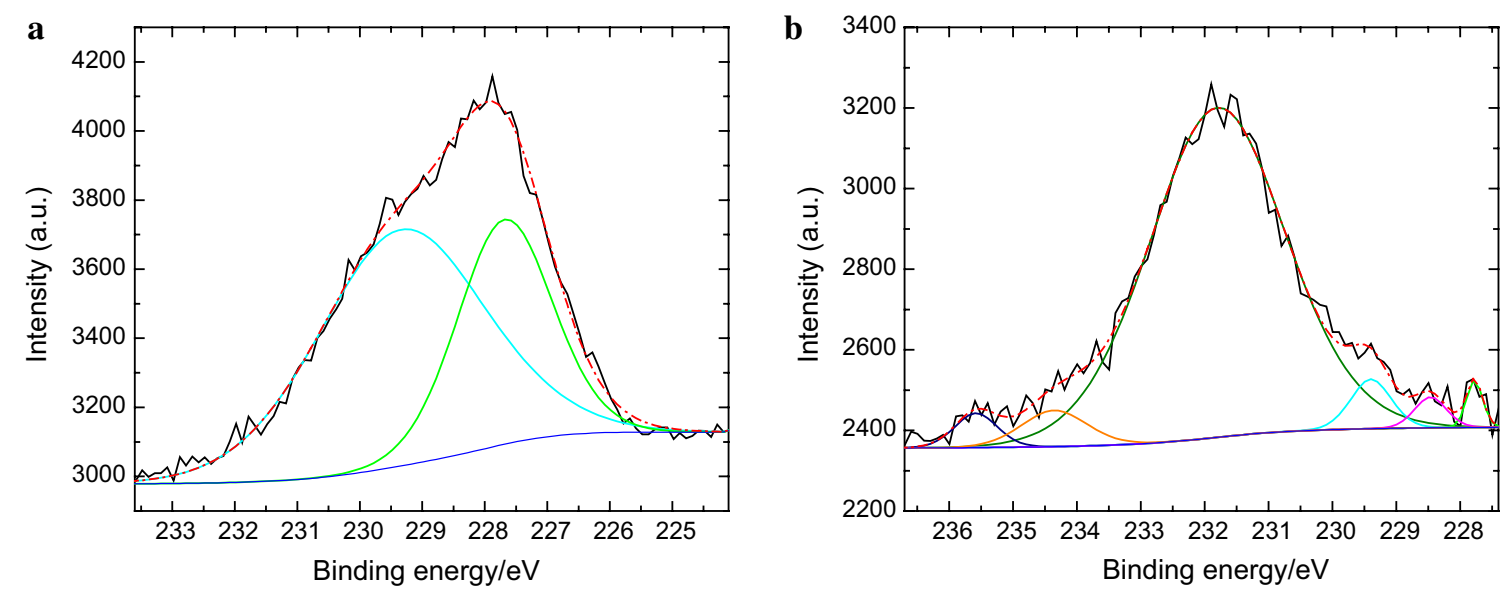

Fig. 7 High-resolution XPS spectra of S 2 s region of a freshly prepared thiol-terminated silicon surface (a) and a PFDT coated silicon surface after TCEP.HCl treatment (b). The black line is the raw data; colored lines denote individual fit components 


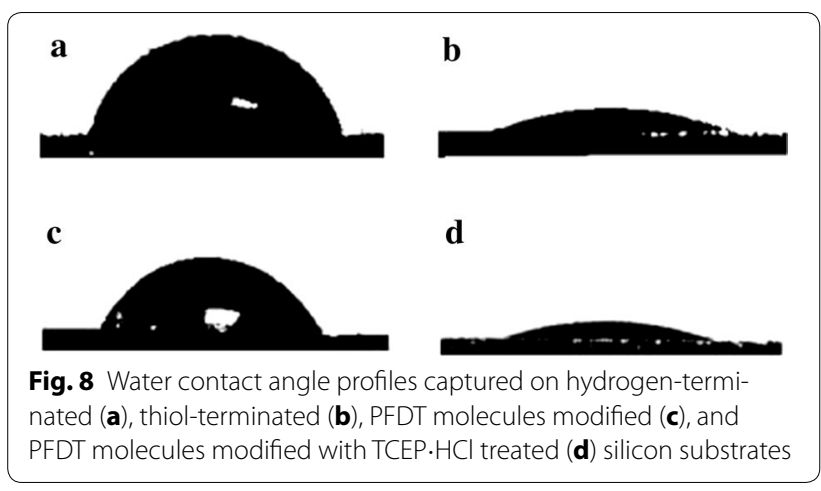

The surface wettability transformation from $78.4 \pm 1.1^{\circ}$ to $23.6 \pm 1.2^{\circ}$ can be attributed to the hydrogen bonds formed between thiol groups on thiolated silicon substrate and water molecules. After chemical modification with PFDT, the water contact angle was changed to about $70.0 \pm 1.9^{\circ}$. This can be attributed to the introduction of the low surface energy of PFDT. After the TCEP.HCl treatment, the water contact angle was changed to about $22.1 \pm 1.6^{\circ}$ (Fig. $8 \mathrm{~d}$ ), implying the release of the PFDT layer from the surface.

Then, PFDT modified Si substrate with TCEP. HCl treatment was incubated in $100 \mathrm{mmol} \mathrm{L}^{-1}$ PFDT anhydrous ethanol solution again for $2 \mathrm{~h}$. After being thoroughly rinsed with anhydrous ethanol by sonicating, the XPS result (Fig. 9) presents a F1s peak at $289 \mathrm{eV}$ with weak intensity. This weak F1s peak indicates that the surface was barely modified by PFDT molecules which correspond to the loss of surface-bound thiol groups due to oxidation.

The antifouling performance of the PFDT modified silicon substrate with TCEP. $\mathrm{HCl}$ treatment was investigated

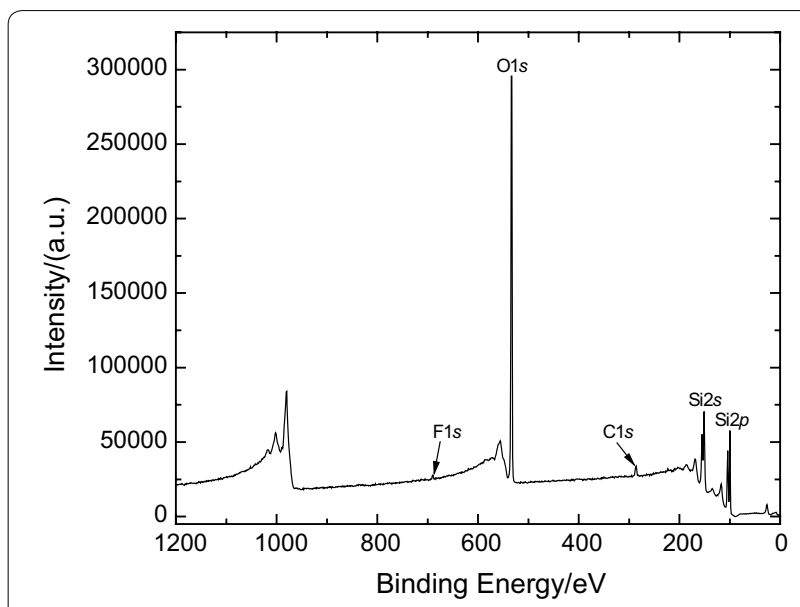

Fig. 9 XPS survey spectrum of silicon surface prepared by first being modified with PFDT molecules, then treated with TCEP.HCI solution, and finally immersed in PFDT anhydrous ethanol solution for $2 \mathrm{~h}$ by following the procedures described previously. After 1 week of incubation in a $B$. braunii culture, the density of cells attached to the surface (PFDT modified silicon substrate with TCEP. $\mathrm{HCl}$ treatment) observed by microscope (Fig. 10) was greater than that on the PFDT modified silicon substrate without TCEP. $\mathrm{HCl}$ treatment. However, the cell density on the PFDT modified silicon substrate with TCEP. $\mathrm{HCl}$ treatment was lower than that on the Piranha solution cleaned silicon substrate. B. braunii cell clusters, which were formed on Piranha solution cleaned silicon substrate, were not observed on PFDT modified silicon substrate with TCEP. $\mathrm{HCl}$ treatment.

In order to evaluate the effect of TCEP. $\mathrm{HCl}$ on the attachment of $B$. braunii, a control experiment was conducted. A Piranha solution cleaned silicon substrate with B. braunii clusters (sample in Fig. 2a) was incubated into Bristol medium containing $10 \mathrm{mM}$ TCEP. $\mathrm{HCl}$ for $1 \mathrm{~h}$. From the microscope image (Fig. 11), the attachment of B. braunii is similar to that of Fig. 2a. This result indicates TCEP. $\mathrm{HCl}$ had no effect on the attachment of B. braunii.

\section{Conclusion}

In summary, PFDT molecules were integrated onto thiolterminated silicon substrate through the formation of disulfide bonds. The PFDT modified silicon substrate appeared to possess, to some extent, a micro-organism resistant property. However, as the time for the immersion test increased, the overall $B$. braunii cell density on the PFDT modified silicon substrate increased indicating its antifouling property cannot last forever. It was found that the adhered $B$. braunii on PFDT modified silicon substrate can be removed by applying TCEP. $\mathrm{HCl}$ solution. TCEP. $\mathrm{HCl}$ serves as a reducing reagent and can therefore break the disulfide bonds and detach the PFDT

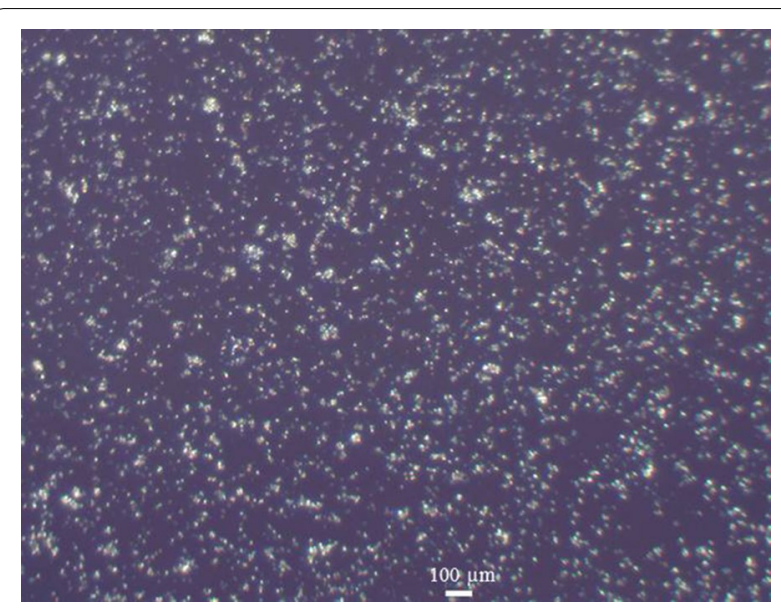

Fig. 10 Representative microscope image of PFDT molecules modified silicon surface with TCEP. HCl solution treatment after immersion test in B. braunii culture for 1 week 


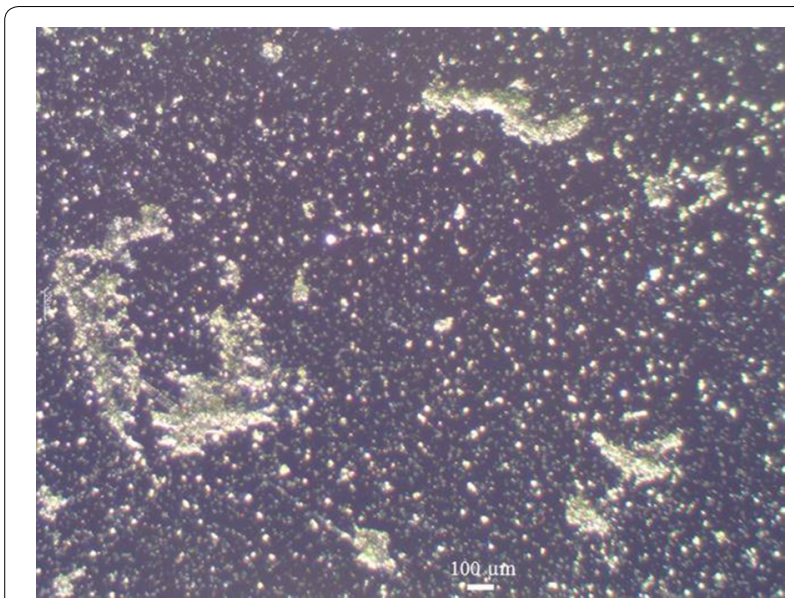

Fig. 11 Representative microscope image of Piranha solution cleaned silicon surface which was first incubated in B. braunii culture at room temperature for 1 week, and then incubated in Bristol medium containing $10 \mathrm{mM}$ TCEP. $\mathrm{HCl}$ at room temperature for $1 \mathrm{~h}$

coating, along with the $B$. braunii cells adhered on it. This presented approach provides a rational design for removing antifouling coating that becomes aged, all without damaging the original substrate.

\section{Additional file}

Additional file 1: Figure S1. XPS survey spectrum of PFDT molecules modified Si surface. Figure S2. Optical density of B. braunii culture at $660 \mathrm{~nm}$.

\section{Abbreviations}

PFDT: 1H,1H,2H,2H-perfluorodecanethiol; DMF: N,N-dimethylformamide; XPS: $X$-ray photoelectron spectroscopy; TCEP.HCl: tris(2-carboxyethyl) phosphine hydrochloride; LB: Luria-Bertani; E. coli: Escherichia coli; B. braunii: Botryococcus braunii.

\section{Authors' contributions}

All authors carried out the experiments and the writing of the manuscript. All authors read and approved the manuscript.

\section{Author details}

${ }^{1}$ College of Biotechnology, Southwest University, Chongqing 400715, China. 2 Department of Chemistry, Eastern Kentucky University, 521 Lancaster Ave, Richmond, KY 40475, USA. ${ }^{3}$ Department of Mathematics, Sciences and Technology, Paine College, Augusta, GA 30901, USA. ${ }^{4}$ Key Laboratory of Mariculture and Enhancement of Zhejiang Province, Marine Fishery Institute of Zhejiang Province, Zhoushan 316021, China.

\section{Acknowledgements}

Xiaoning Zhang gratefully acknowledges the financial support from the National Science Foundation (HRD-1505197) and a Start-up Fund of Southwest University grant (SWU1 17036). We thank Dr. Yinan Wei of University of Kentucky for providing E. coli as a gift for this research.

\section{Competing interests}

The authors declare that they have no competing interests.

Ethics approval and consent to participate Not applicable.

\section{Publisher's Note}

Springer Nature remains neutral with regard to jurisdictional claims in published maps and institutional affiliations.

Received: 5 October 2017 Accepted: 1 February 2018

Published online: 07 February 2018

\section{References}

1. Magin CM, Cooper SP, Brennan AB (2010) Non-toxic antifouling strategies. Mater Today 13(4):36-44

2. Hydrex NV (2010) Ship hull performance in the post-TBT Era. The Hydrex Group, Belgium. http://ec.europa.eu/environment/life/project/Projects/ index.cfm?fuseaction=home.showFile\&rep=file\&fil=ECOTEC_Ship_Hull_ Performance.pdf

3. Schultz MP (2007) Effects of coating roughness and biofouling on ship resistance and powering. Biofouling 23(5):331-341

4. Schultz MP, Bendick JA, Holm ER, Hertel WM (2011) Economic impact of biofouling on a naval surface ship. Biofouling 27(1):87-98

5. Briand J-F (2009) Marine antifouling laboratory bioassays: an overview of their diversity. Biofouling 25(4):297-311

6. Zargiel KA, Coogan JS, Swain GW (2011) Diatom community structure on commercially available ship hull coatings. Biofouling 27(9):955-965

7. Sharma S, Johnson RW, Desai TA (2004) XPS and AFM analysis of antifouling PEG interfaces for microfabricated silicon biosensors. Biosens Bioelectron 20(2):227-239

8. Li M, Neoh KG, Xu LQ, Wang R, Kang ET, Lau T (2012) Surface modification of silicone for biomedical applications requiring long-term antibacterial, antifouling, and hemocompatible properties. Langmuir Acs I Surf Colloids 28(51):16408-16422

9. Zhang X, Brodus D, Hollimon V, Hu H (2017) A brief review of recent developments in the designs that prevent bio-fouling on silicon and silicon-based materials. Chem Cent J 11(1):18

10. Rana D, Matsuura T (2010) Surface modifications for antifouling membranes. Chem Rev 110(4):2448-2471

11. Zhang X-N, Hollimon V, Brodus D (2016) A method for attaching thiol groups directly on a silicon (111) substrate. Acta Phys Chim Sin 32(9):2364-2368

12. Lamour G, Hamraoui A, Buvailo A, Xing Y, Keuleyan S, Prakash V et al (2010) Contact angle measurements using a simplified experimental setup. J Chem Educ 87(12):1403-1407

13. Drelich J, Miller JD, Hupka J (1993) The effect of drop size on contact angle over a wide range of drop volumes. J Colloid Interface Sci 155(2):379-385

14. Salwiczek M, Qu Y, Gardiner J, Strugnell RA, Lithgow T, Mclean KM et al (2014) Emerging rules for effective antimicrobial coatings. Trends Biotechnol 32(2):82-90

15. Tang J, Han Y, Chen H, Lin Q (2016) Bottom-Up fabrication of PEG brush on poly(dimethylsiloxane) for antifouling surface construction. Int J Polymer Sci 2016:5

16. Cordeiro A, Nitschke M, Janke A, Helbig R, D'Souza F, Donnelly G et al (2009) Fluorination of poly (dimethylsiloxane) surfaces by low pressure CF4 plasma-physicochemical and antifouling properties. Express Polymer Lett 3(2):70-83

17. Li Y, SuY, Zhao X, Zhang R, Zhao J, Fan X et al (2014) Surface fluorination of polyamide nanofiltration membrane for enhanced antifouling property. J Membr Sci 455:15-23

18. Velleman L, Shapter JG, Losic D (2009) Gold nanotube membranes functionalised with fluorinated thiols for selective molecular transport. J Membr Sci 328(1-2):121-126

19. Bukhtiyarova GA, Bukhtiyarov VI, Sakaeva NS, Kaichev VV, Zolotovskii BP (2000) XPS study of the silica-supported Fe-containing catalysts for deep or partial $\mathrm{H}_{2} \mathrm{~S}$ oxidation. J Mol Catal A Chem 158(1):251-255

20. Madaan N, Romriell N, Tuscano J, Schlaad H, Linford MR (2015) Introduc tion of thiol moieties, including their thiol-ene reactions and air oxidation, onto polyelectrolyte multilayer substrates. J Colloid Interface Sci 459:199-205 